\title{
Mobile Commerce: A New Frontier
}

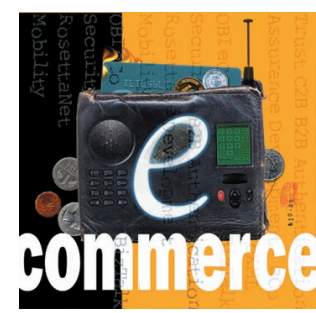

\begin{abstract}
As wireless networks grow, mobile electronic commerce will soon follow. How must wireless technologies change to support this growing market? The authors propose a mobile commerce framework to accommodate this new development.
\end{abstract}

Upkar Varshney Georgia State University

Ronald J. Vetter

University of North

Carolina at Wilmington

Ravi

Kalakota hsupply.com

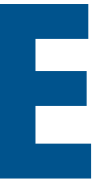
lectronic commerce continues to see phenomenal growth, but so far most e-commerce development involves wired infrastructures. We believe emerging wireless and mobile networks will provide new avenues for growth, creating new opportunities in mobile commerce.

The exponential growth of wireless and mobile networks has brought vast changes in mobile devices, middleware development, standards and network implementation, and user acceptance. More than 350 million mobile devices are in use worldwide, 80 million of them in the United States. ${ }^{1}$ Analysts expect this number to rise to 1 billion in the next few years, exceeding the total number of computing devices by several fold. ${ }^{2}$

According to the GartnerGroup, a market research firm, by 2004 at least 40 percent of consumer-to-business e-commerce will come from smart phones using the wireless application protocol (WAP). ${ }^{3}$ Based on a study by the Wireless Data and Computing Service, a division of Strategy Analytics, the annual mobile commerce market may rise to $\$ 200$ billion by 2004 .

We believe the next phase of electronic business growth will be in wireless and mobile commerce. To help future applications and technologies handle this growth, we propose a framework for mobile commerce.

\section{MOBILE COMMERCE FRAMEWORK}

Figure 1 shows our framework for mobile commerce, which lets companies strategize and create mobile commerce applications. The framework defines several functional layers, simplifying the design and development so that different parties - vendors, providers, designers, and so on-can address individual layers. By using this framework, a single entity is not forced to do everything to build mobile commerce systems, but can build on the functionalities provided by others. The framework includes a user plane with four levels:
- Applications. Many new applications are becoming possible, and many existing e-commerce applications can be modified for a mobile environment.

- User infrastructure. The design of new mobile commerce applications should consider the capabilities of the user infrastructure-the mobile devices.

- Wireless middleware. With its ability to hide the underlying network's details from applications while providing a uniform and easy-to-use interface, middleware is extremely important for developing new mobile commerce applications.

- Network infrastructure. In mobile commerce, service quality primarily depends on network resources and capabilities.

The framework also provides a developer-provider plane, which addresses the different needs and views of application developers, content providers, and service providers.

\section{EMERGING APPLICATIONS}

With an unlimited number of potential mobile commerce applications, the best way to understand them is to classify them, as in Table 1. Of particular interest are mobile inventory management, product location, and proactive service management.

\section{Mobile inventory management}

Mobile inventory management tracks the location of goods, services, and possibly even people so that providers can determine delivery times, thus improving customer service and enhancing a company's competitive edge. In rolling inventory management, multiple trucks carry large amounts of inventory. When a store needs certain items, it can locate a nearby truck carrying them (Figure 2), obtain just-in-time delivery, and thus reduce inventory space and cost. 
The system's wireless network would embed inexpensive radio-wave and microwave devices (chips) somewhere in the delivery truck to track goods. Since satellite signals may not work well inside a truck, each vehicle would have a separate onboard wireless LAN for intra-truck communication and tracking.

Another example of mobile inventory management is just-in-time delivery of components to assembly plants. After receiving a wireless signal from components reaching the assembly line or from a device on the assembly line itself, a supplier could begin shipping the required number of components at a certain speed. If the components should be delayed, the supplier can send signals to the assembly line to adjust its speed to match the revised arrival time.

Mobile inventory management's success depends on cost, wireless infrastructure reliability, and users' level of comfort with the new technology. Potential users include shipping companies, assembly plants, airlines and other mass-transit companies, and supermarket chains. Most of these industries are already increasing their use of wireless technology.

\section{Product location}

Consumers find an item with certain specifications in a particular area. The search may turn up several businesses selling the item or something similar. The product location service could alleviate the need to visit several stores to find a particular item or to comparison shop. Instead, consumers could use a mobile device like a PalmPilot, Nokia Communicator, or Net Phone to access a database of products and the stores that sell them. The resulting list could also provide distances from the user's location to the stores. When multiple vendors carry the item, they could compete by offering instant discounts.

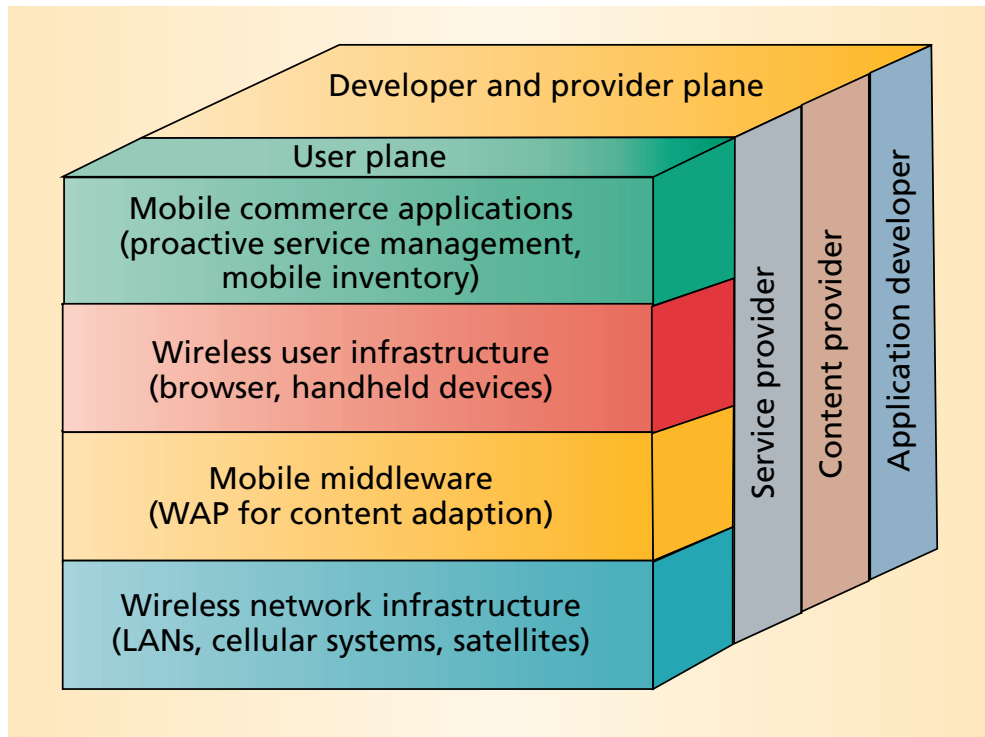

Figure 1. The proposed framework for mobile commerce showing a user plane with four levels and a developer-provider plane with three.

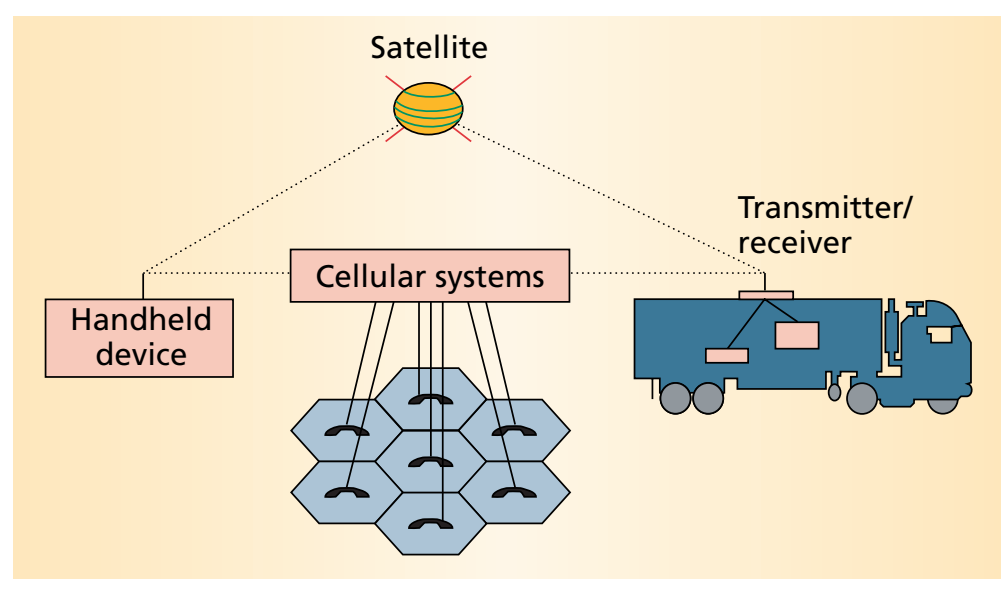

Figure 2. Location tracking of goods. A handheld or other computing device can track the location of goods to provide rolling inventory management. Different wireless networks may be necessary, depending on the size of the objects and the surroundings.

Table 1. Classes of mobile commerce applications.

\begin{tabular}{|c|c|}
\hline Class of wireless applications & Examples \\
\hline Mobile inventory management & $\begin{array}{l}\text { Tracking the location of goods and services, such as boxes, packets, troops, } \\
\text { or cattle }\end{array}$ \\
\hline Product location & Locating certain items, such as TVs, VCRs, or cars \\
\hline Proactive service management & $\begin{array}{l}\text { Transmitting information about aging components, such as automobile parts, } \\
\text { to vendors }\end{array}$ \\
\hline Wireless reengineering & Improving business services, such as claim adjustments or insurance \\
\hline Mobile auction and reverse auction & Offering, selling, and bidding \\
\hline Mobile entertainment services & Providing services such as video on demand \\
\hline Mobile office & $\begin{array}{l}\text { Providing services for businesspeople, such as traffic jam reports, airport and } \\
\text { flight information, vacation reservations, and procurement of products and } \\
\text { services }\end{array}$ \\
\hline Mobile distance education & Offering classes using streaming audio and video \\
\hline Wireless data center & Providing downloadable information from data warehouses \\
\hline Mobile music and music on demand & Allowing downloading and storing of music from the Internet \\
\hline
\end{tabular}




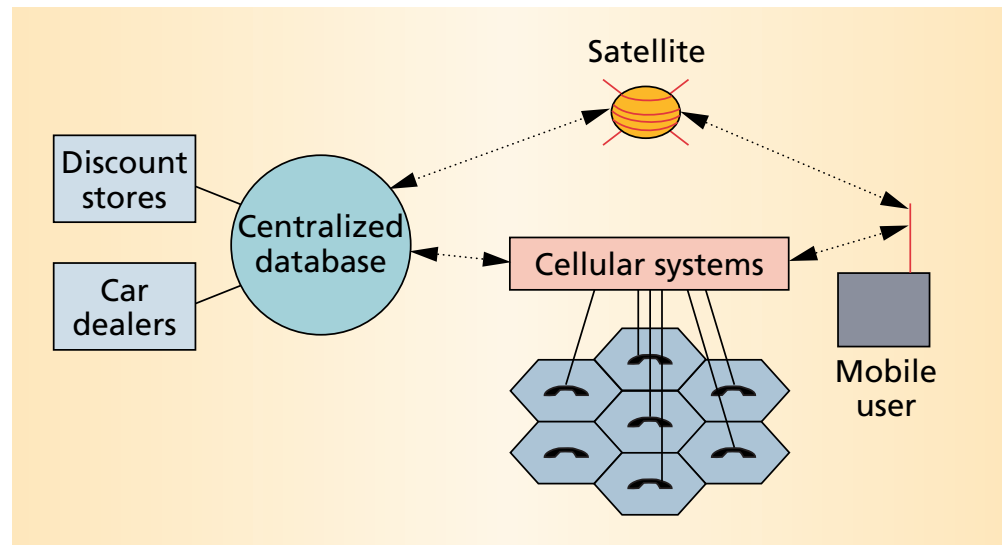

Figure 3. Product location. A mobile user searches for a certain item by using wireless networks and a database. The user can specify the search range, or the database can assume the immediate neighborhood of the user's current location.

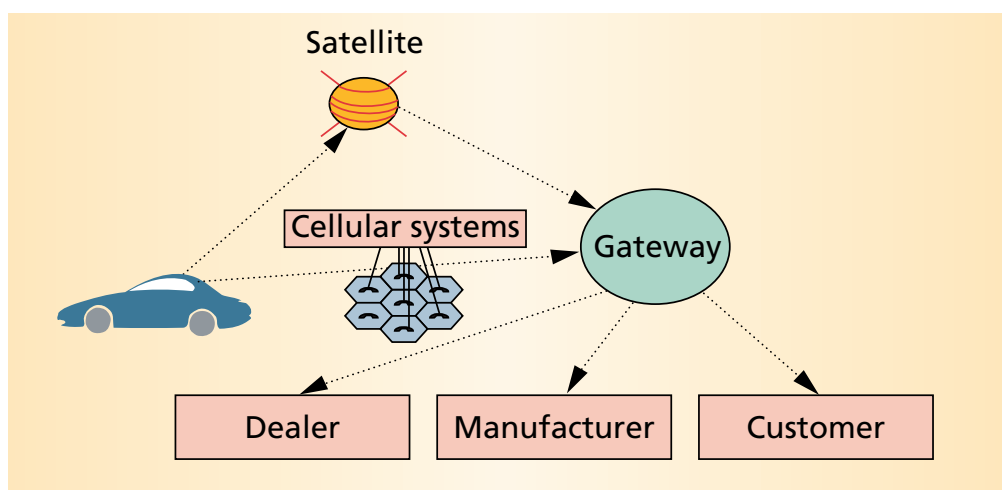

Figure 4. Smart sensors on an automobile relay information on component conditions to appropriate parties. A central access point (gateway) lets such information travel over wireless networks to a single location, from which it goes to specific parties. Manufacturers can alert customers about levels of wear and tear in components and improve the quality of future products. Dealers can market the needed services to customers and better plan their inventory.

As shown in Figure 3, the mobile user sends a query to a centralized database, which interfaces with several vendors to determine a product's availability and price. The database searches each company's inventory record system, but differences in coding may require that everyone use a uniform product-naming system. If traffic becomes a problem, the database can ship codes rather than data. Sending these product codes instead of detailed product information may reduce the amount of traffic over wireless networks.

Two factors to consider in this service are how the database owner will price its services to vendors and how to ensure the accuracy of information on availability and price. A service provider could deploy multiple cooperating and negotiating software agents to conduct various transactions at different places.

\section{Proactive service management}

Applications collect information on user needs and then signal vendors to provide services. For example, an application may collect information on aging components of automobiles, which vendors could use to solicit more business or to better manage component invento- ries. Dealers could use the information to compete more effectively by offering discounts or lower rates. Such detailed information lets them target their marketing to users who may need these services in the near future.

Manufacturers could collect and analyze such information to improve the design and manufacturing of their products. Also, they could offer proactive service management as part of the automobile warranty or as an add-on service, alerting owners when parts need replacing. By improving the general condition of automobiles, the service might reduce breakdowns, traffic jams, and even fatalities.

As Figure 4 shows, smart sensors on the automobile would keep track of component wear and tear and relay the information to appropriate parties using a radio, microwave, or satellite system.

Implementing such a system would require resolving privacy and security issues, ensuring information reliability, and funding initial costs. However, car dealers, repair shops, and car owners would be interested in such a system. In the future, police officers could use it to increase the safety of everyone on the road through proactive law enforcement.

\section{Mobile auction, entertainment, and other services}

While mobile, people still want to handle business or enjoy entertainment. Mobile devices and wireless networks can provide auctions, video on demand, and information services such as enhanced quality news. People would require such technology as appropriate mobile devices, mobile middleware, and high-bandwidth wireless networks - such as emerging Low-Earth-Orbit satellites or third-generation wireless networks-or video compression techniques.

Connectivity is important because disconnects seriously affect the quality of entertainment and information services. For auctions, frequent disconnection would undermine the service's usefulness unless the service provider or carrier could guarantee that the auction's state would be maintained until the user reconnects, thus preventing any user losses.

\section{WIRELESS USER INFRASTRUCTURE}

To realize these applications, we need several functional components, starting with a mobile device with sufficient memory, an appropriate display, and communications functionalities. Several suitable models are now available, such as the PalmPilot-a personal digital assistant (PDA) with a wireless modem-and the Nokia Communicator-a mobile phone with computing functions. These devices are oriented toward either communication or computing, but in the near future these distinctions could disappear as intelligent mobile devices evolve.

As these devices gain more functions and grow in storage and processing capabilities, they'll need an 
Table 2. Operating systems for handheld and smaller devices.

\begin{tabular}{|c|c|c|c|c|}
\hline Mobile OS & Vendor & Footprint & Deployed in & Comments \\
\hline Palm OS & $\begin{array}{l}\text { Palm Computing } \\
\text { (3Com) }\end{array}$ & 300 Kbytes & $\begin{array}{l}\text { Palm Pilot PdQ } \\
\text { (Qualcomm) }\end{array}$ & Simple and easy to use \\
\hline GEOS 3.0 & Geoworks & 500 Kbytes & Nokia 9000 Communicator & Fast \\
\hline Windows CE 2.0 & Microsoft & 2 Mbytes & $\begin{array}{l}\text { Nino, MobilePro, and other } \\
\text { palm-size PCs }\end{array}$ & $\begin{array}{l}\text { Slow, but } \\
\text { more features }\end{array}$ \\
\hline OS-9 & Microware & 300 Kbytes & In-car navigation systems & Extremely fast \\
\hline Epoc32 & $\begin{array}{l}\text { Symbian (Ericsson, } \\
\text { Motorola, Nokia) }\end{array}$ & 1 Mbyte & $\begin{array}{l}\text { Synergy (a GSM } \\
\text { attachment unit) }\end{array}$ & $\begin{array}{l}\text { Built-in tolerance for } \\
\text { hardware failure }\end{array}$ \\
\hline
\end{tabular}

operating system to manage resources. A general-purpose OS is not suitable for these devices because of their real-time requirements, processing power, limited memory, small screen size, and typical applications - such as voice. These devices need an OS with a small footprint and reduced storage needs, such as those shown in Table 2. Nearly all OS vendors have attracted developers of applications for handheld and smaller devices. Because Unix has been used widely on the Internet and in other computing environments, a stripped-down version requiring a smaller footprint may become important for mobile applications.

\section{WIRELESS AND MOBILE MIDDLEWARE}

Middleware unites different applications, tools, networks, and technologies, giving users a common interface. Mobile middleware is an enabling layer of software to connect e-commerce applications with different mobile networks and operating systems without introducing mobility awareness - the need to adjust to wide variations in bandwidth and resulting delays, and changes in user location-in the applications, as Figure 5 shows.

Middleware gives applications better response times and far better reliability. Typically, middleware uses optimization techniques, such as header compression, delayed acknowledgments, and concatenation of several smaller packets into one to reduce wireless network traffic. Some middleware supports intelligent restarts, which take the user to the break point after disconnection instead of back to the beginning. Middleware, however, does introduce additional complexity and significant initial cost.

\section{ExpressQ}

ExpressQ from Nettech (www.nettechRF.com) is a mobile-messaging middleware product that lets developers extend their non-IP applications to mobile users. It stores messages when mobile users are out of network range and forwards them later when users are in range. ExpressQ uses logical name addressing to allow network and device independence, supports several wireless networks, provides multiple application programming interfaces (APIs) for developers, and lets mobile devices run different operating systems.

\section{Wireless application protoco}

Using a common set of applications and protocols, WAP facilitates interoperability among different wire-

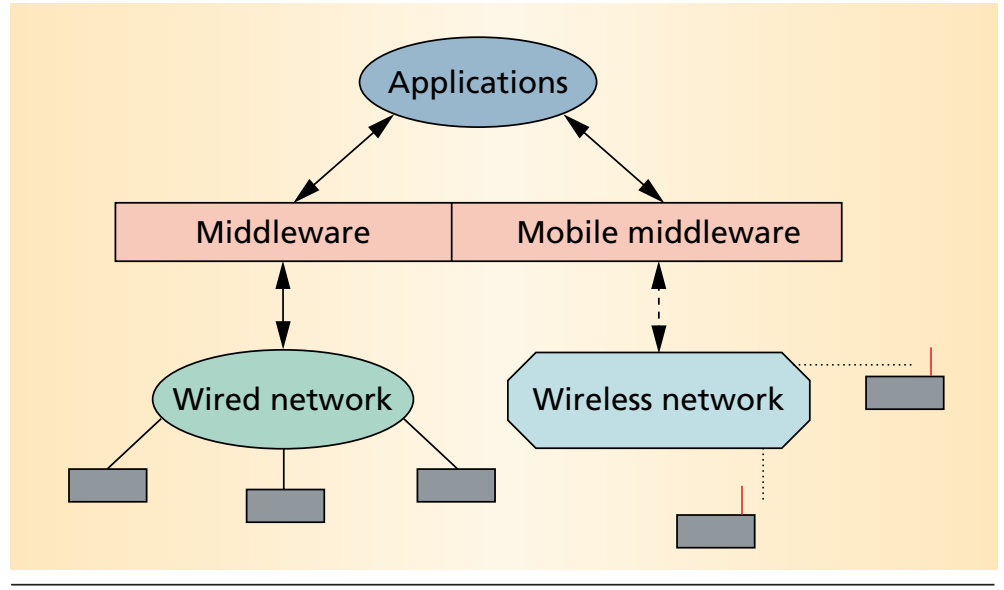

Figure 5. Mobile middleware for application and content adaptation. Mobile middleware can hide the differences in applications from a mobile user. It can also reduce the content size and format to better adapt to the inherent characteristics of wireless networks and the limitations of mobile devices.

less networks, devices, and applications. WAP uses a microbrowser as the client software and supports text, graphics, and standard Web content. In the near future, it will support streaming media, such as video.

As Figure 6 shows, a gateway acts as a proxy server for a mobile client, translating WAP requests to protocols employed by the information server on the other side. Encoders translate the content coming from the server into compact formats to reduce the size of data over the wireless network. This infrastructure lets mobile users access a wide variety of content and lets application developers, using proven and existing technologies, build applications that run on a large base of mobile terminals.

\section{Emerging W3C standards}

In the future, Web content will need to be accessible everywhere-from PCs, to TVs, to PDAs, to cellular phones. Therefore, the World Wide Web Consortium (W3C) has developed several open recommendations for extending existing Internet standards so that wireless devices can fully access the Web and its information base. With the rapid introduction of new kinds of Web browsers (such as digital TVs and various handheld devices) and new types of Web page input (such as microphones, cameras, scanners, and pen-based devices), improved document markup and style sheet support are clearly needed. 


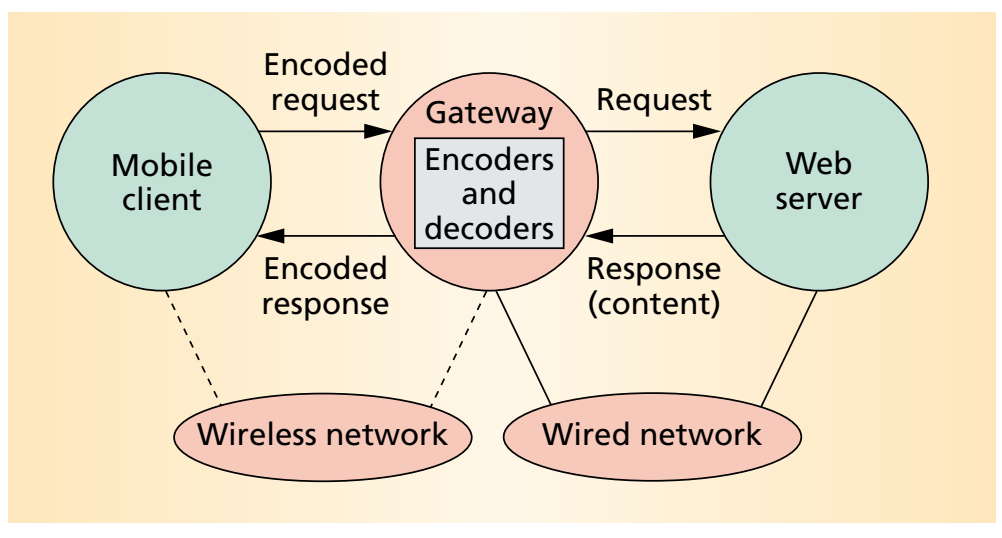

Figure 6. WAP architecture. WAP uses a proxy gateway to translate requests from mobile clients to a Web server and to adapt contents from the server to the mobile clients. WAP encrypts the communication between the mobile client and the gateway to provide some level of security for transactions.

The W3C has devised several recommendations to allow Web device independence, content reuse, and network-friendly encoding:

- the Extensible Markup Language (XML) for richer semantic information;

- improved cascading style sheets and Extensible Stylesheet Language to further separate content from presentation; and

- a document object model defining a languageindependent API that applications can use to access and modify HTML and XML documents' structure, content, and style.

These W3C specifications, along with the WAP specifications, will enable a wide range of wireless networking applications.

\section{WIRELESS NETWORKING INFRASTRUCTURE}

Besides mobile devices and middleware, mobile commerce applications depend on networking support. Table 3 shows some of the network issues.

Two of the most import factors, of course, are bit rate and coverage. Developers are now standardizing the third generation of wireless networks, which will support several megabits per second. ${ }^{5}$ Because many mobile commerce transactions require small messages, mobile commerce applications may use the short messaging service of the European GSM (Groupe Spécial Mobile) or other networks.

Another important factor is support for multicasting-communications among a selected group of users-which may be necessary for group-oriented applications such as electronic auctions. Wireless and mobile networks are now considering support for multicasting. ${ }^{6}$

Although individual applications have their own special requirements, electronic commerce in general also needs

- wireless quality of service, which may affect the performance of mobile commerce applications in terms of delay, loss, and other quality attributes;

- location management, an integral part of many mobile commerce applications, especially those involving location or product searches;

- reliable and survivable wireless networks so that users can access mobile commerce applications, even under varying degrees of network failure; and

- roaming across multiple heterogeneous networks so that users can access mobile commerce applications from anywhere, even when the coverage of a single wireless network is spotty.

Table 3. A comparison of several mobile and wireless networks.

\begin{tabular}{|c|c|c|c|c|c|c|}
\hline Issue & $\begin{array}{l}\text { Wireless } \\
\text { LANs }\end{array}$ & $\begin{array}{l}\text { Wireless } \\
\text { loops }\end{array}$ & $\begin{array}{l}\text { Cellular/ } \\
\text { PCS }\end{array}$ & Mobile IP & $\begin{array}{l}\text { Wireless } \\
\text { ATM }\end{array}$ & Satellites \\
\hline Coverage & Local area & $\begin{array}{l}\text { Local }^{*} \text { or } \\
\text { metropolitan }\end{array}$ & Metropolitan & Wide area & Wide area & Wide area \\
\hline $\begin{array}{l}\text { User } \\
\text { bandwidth }\end{array}$ & 1 to $20 \mathrm{Mbps}$ & 1 to $20 \mathrm{Mbps}$ & $19.2 \mathrm{Kbps}$ & $\begin{array}{l}\text { Network } \\
\text { dependent ** }\end{array}$ & 1 to $20 \mathrm{Mbps}$ & $\begin{array}{l}19.2 \text { Kbps to } \\
\text { several Mbps*** }\end{array}$ \\
\hline Application & Data and voice & Data and voice & Data and voice & Data and voice & All & Data and voice \\
\hline Limitations & Limited area & Interference & Bandwidth & $\begin{array}{l}\text { Limited } \\
\text { applications }\end{array}$ & Cost & Initial cost \\
\hline Status & In use & Emerging & In use & Emerging & Emerging & Emerging \\
\hline $\begin{array}{ll}* & \text { Dependin } \\
* * & \text { Depends } \\
* & \text { Higher lin }\end{array}$ & $\begin{array}{l}\mathrm{n} \text { the underlyin } \\
\text { the underlying } \\
\text { for satellites lik }\end{array}$ & $\begin{array}{l}\text { technology (su } \\
\text { vireless network. } \\
\text { Teledesic. }\end{array}$ & as $3-10$ miles & $\overline{M D S)}$ & & \\
\hline
\end{tabular}


Table 4. Important issues for application developers.

\begin{tabular}{|c|c|}
\hline Issues & Comments \\
\hline \multirow{5}{*}{$\begin{array}{l}\text { Network processing and } \\
\text { storage requirements }\end{array}$} & Understand bandwidth and delay requirements for real-time and non-real-time applications \\
\hline & $\begin{array}{l}\text { Understand mobile device capabilities and find ways to work around some limitations of } \\
\text { mobile devices }\end{array}$ \\
\hline & Support disconnected operations \\
\hline & Support multicasting for group communications \\
\hline & Consider using asymmetric processing and storage \\
\hline \multirow[t]{5}{*}{ Application development } & $\begin{array}{l}\text { Consider using an existing software development kit for simulation of environment where } \\
\text { application will be used }\end{array}$ \\
\hline & Take into account the maximum number of simultaneous users \\
\hline & Identify any restrictions on the size of application code \\
\hline & Support secure transactions \\
\hline & Support fixed users \\
\hline \multirow{4}{*}{$\begin{array}{l}\text { Compatibility } \\
\text { and interoperability }\end{array}$} & Provide independence from the underlying wireless access technologies \\
\hline & Provide independence from the device functionalities \\
\hline & Consider IP interoperability \\
\hline & Consider WAP or at least provide compatibility with WAP \\
\hline \multirow[t]{5}{*}{ Desirable features } & Consider the support for intermittent connectivity \\
\hline & Consider introducing adaptivity to the user and network environment \\
\hline & Support atomic transactions \\
\hline & If possible, introduce the ability to be easily upgraded \\
\hline & Support user-specified features invocation \\
\hline
\end{tabular}

One issue will be differences between European and American wireless services, including differences in infrastructure, pricing, level of regulation, standards agreements, and business culture. While European countries have agreed to a common standard, the United States still suffers from multiple and incompatible standards. As a result, by 2003, mobile commerce will amount to $\$ 23.6$ billion in Europe ${ }^{7}$ but only $\$ 3$ billion in the United States.

\section{ISSUES FOR DEVELOPERS}

Table 4 outlines issues that developers must address to successfully define, design, and implement the necessary hardware and software infrastructure for mobile commerce.

Achieving application independence from device and wireless technologies is a major issue, but designing for independence will likely increase the actual program code. To increase usability across networks and devices, these applications can assume only basic hardware functionalities, but this approach leads to less flexible implementations.

\section{A CASE STUDY}

To better understand our framework, let's look at Phone.com's proprietary framework, which provides a complete solution to wireless Internet access (http://www. phone.com/products). The company's microbrowser, UP.Browser, presents both graphics and text, accepts user choices, and interprets alphanumeric data entry, letting users interact with Web-based applications. The browser can also alert users when a service or application pushes a Web page or other data to them. One concern with this browser is its compatibility with other products.

As Figure 7 shows, a gateway server, UP.Link, provides middleware functionality. It gives mobile users access to the Internet by providing protocol translation and optimization, security, and activity tracking. Phone.com has implemented UP.Link in a proprietary

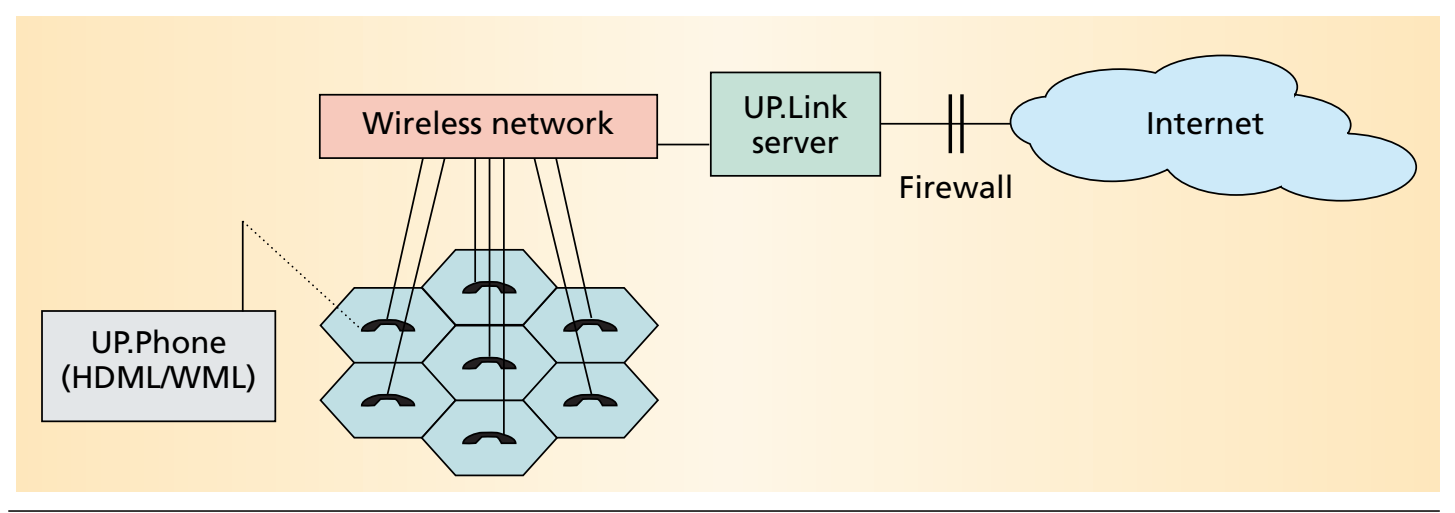

Figure 7. Phone.com's solution to wireless Internet access includes a microbrowser (UP.Browser) on a user device (UP.Phone), a wireless network, and a link server. Some simple applications use this configuration. 
manner, so interoperability with other implementations will be a major issue.

Phone.com provides many applications, such as UP.Smart, a suite of portable smart-phone applications with popular PDA features. Another application is UP.Mail, which lets mobile phone users receive and send e-mail from their wireless phones using their existing e-mail accounts. If they don't have an e-mail account, UP.Mail provides one. Most applications handle common tasks like e-mail and personal organization but do not allow sophisticated two-way group interactions needed for most mobile commerce transactions.

To support developers, Phone.com makes its software development kit, UP.SDK, freely available. Web developers can create applications based on Wireless Markup Language and Handheld Device Markup Language (HDML) - Phone.com's proprietary language. UP.SDK includes UP.Simulator to simulate a device enabled for UP.Browser. Two problems with the kit are the use of a proprietary language for development and lack of support for highly sophisticated application development, both of which are necessary for implementing sophisticated mobile commerce applications.

The UP solution demonstrates that a product based on a proprietary framework is implemented in an ad hoc fashion. Future products must conform to a standard and open framework — such as the one presented here-to interoperate in a seamless fashion.

$\mathbf{0}$ ne factor we have not addressed is user trust in mobile commerce applications, which will play a crucial role in acceptance and widespread deployment. Trust will grow if we increase network reliability and redundancy, improve security, and support atomic transactions - transactions in which all or no steps are executed-very important for mobile commerce transactions in case of disconnection.

Creating mobile commerce applications requires a unique breadth of knowledge. The development of the next frontier of e-commerce will require the active participation of economists, computer and telecommunications experts, social scientists, and business strategists. Industry leaders must promote and advocate an open, standards-based framework for mobile electronic commerce. We hope this article provides technologists and users alike with a better understanding of the trends, performance trade-offs, and system interdependencies involved at every layerapplications, user infrastructure, wireless middleware, and network infrastructure-of the mobile commerce framework. *

\section{References}

1. Cellular Telecommunications Industry Assoc., "CTIA's Semi-Annual Wireless Industry Survey,” Wow-com, Dec. 1999. http://www.wow-com.com/statsurv/survey.

2. ClickServices.com, "ClickServices.com Unleashes a New Web Portal for Wireless Internet," Wow-com, 25 Feb. 2000, http://www.wow-com.com/newsline/press_release. cfm?press_id=990.

3. D. Haskin, "Analysts: Smart Phones to Lead E-Commerce Explosion,” allNetDevices, 3 Nov. 1999, http:// www.allnetdevices.com/news/9911/991103ecomm/991 103ecomm.html.

4. Strategy Analytics, "Strategy Analytics Forecasts $\$ 200$ Billion Mobile Commerce Market by 2004," Wow-com, 10 Jan. 2000, http://www.wow-com.com/newsline/ press_release.cfm?press_id $=862$.

5. U. Varshney and R. Vetter, "Emerging Mobile and Wireless Networks," Comm. ACM, June 2000, pp.73-81.

6. U. Varshney, "Multicast Communications over Wireless and Mobile Networks," to appear in Comm. ACM.

7. F. Müller-Veerse, Mobile Commerce Report, Durlacher Corp., London, http://www.durlacher.com/downloads/ mcomreport.pdf.

8. M. Oliphant, "The Mobile Phone Meets the Internet," IEEE Spectrum, Aug. 1999, pp. 20-28.

Upkar Varshney is an assistant professor in the Department of Computer Information Systems at Georgia State University. His research interests include wireless and mobile networks, multicasting in wireless networks, and wireless applications. Varshney has a PhD in telecommunications and computer networking from the University of Missouri, Kansas City. Contact him at uvarshney@gsu.edu.

Ronald J.Vetter is a professor and chair of the Department of Computer Science at the University of North Carolina at Wilmington. His research interests include Web-centric computing, multimedia systems, computer networks, and distance education. Vetter has a $\mathrm{PhD}$ in computer science from the University of Minnesota.Contact himatvetterr@uncwil.edu.

Ravi Kalakota is the founder of hsupply.com, a business-to-business e-commerce company. Previously, he was a member of the CIS faculty and the Center for Digital Commerce at Georgia State University. Kalakota has a PhD in information systems from the University of Texas at Austin. Contact him at ravi@hsupply.com. 